\title{
BATCH FABRICATION OF SCANNING MICROSCOPY PROBES FOR THERMAL AND MAGNETIC IMAGING USING STANDARD MICROMACHINING
}

\author{
E. Sarajlic ${ }^{1}$, R. Vermeer ${ }^{2}$, M.Y. Delalande ${ }^{2}$, M.H. Siekman ${ }^{1,2}$, R. Huijink ${ }^{1}$, H. Fujita ${ }^{3}$, L. Abelmann ${ }^{2}$ \\ ${ }^{1}$ SmartTip B.V., THE NETHERLANDS \\ ${ }^{2}$ TST, MESA + Research Institute, University of Twente, THE NETHERLANDS \\ ${ }^{3}$ CIRMM, Institute of Industrial Science, The University of Tokyo, JAPAN
}

\begin{abstract}
We present a process for batch fabrication of a novel scanning microscopy probe for thermal and magnetic imaging using standard micromachining and conventional optical contact lithography. The probe features an AFMtype cantilever with a sharp pyramidal tip composed of four freestanding silicon nitride nanowires coated by conductive material. The nanowires form an electrical cross junction at the apex of the tip, addressable through the electrodes integrated on the cantilever. The cross junction on the tip apex can be utilized to produce heat and detect local temperature changes or to serve as a miniaturized Hall magnetometer enabling, in principle, thermal and magnetic imaging by scanning the probe tip over a surface. We have successfully fabricated a first probe prototype with a nanowire tip composed of $140 \mathrm{~nm}$ thick and $11 \mu \mathrm{m}$ long silicon nitride wires metallized by 6 $\mathrm{nm}$ titan and $30 \mathrm{~nm}$ gold layers. We have experimentally characterized electrical and thermal properties of the probe demonstrating its proper functioning.
\end{abstract}

\section{INTRODUCTION}

Scanning thermal microscopy (SThM) [1-5] and scanning Hall probe microscopy (SHPM) [6-9] are widely applied techniques for the study of micro- and nanoscale thermal and magnetic phenomena. At the heart of these techniques is a modified scanning probe, which consists of an cantilever having a sharp tip with a nanometer-scale electrical junction integrated at its apex. An electrical junction at the tip apex can be utilized to determine thermal and magnetic properties of a sample in addition to its surface topography. In thermal imaging the junction serve as a localized source of heat as well as a miniaturized temperature sensor allowing measurement of spatial distribution of temperature, thermal conductivity and thermal diffusivity. On the other hand, an electrical junction in the form of a cross functions as a miniaturized Hall magnetometer enabling quantitative measurements of the surface magnetic field.

Both thermal and Hall scanning probes can be realized by combining conventional micromachining with multiple level direct-write electron beam nanolithography $[3,4,9]$. Although this method allows batch fabrication of the probes, high cost of an E-beam system and the serial nature of its writing process make this method both expensive and unsuitable for high-volume manufacturing. Furthermore, the fabricated probes have a blunt tip, which is roughly $1 \mu \mathrm{m}$ by $1 \mu \mathrm{m}$ at the flat top [9], giving poor access to the sample surface at small scales. An alternative fabrication method is based on crafting of a micromachined piezoresistive AFM cantilever by focused electron beam deposition of platinum [5]. This method allows fabrication of the scanning probes with a tip made up of freestanding conductive nanowires. A relatively sharp tip with low thermal mass makes these hand-crafted probes particularly suitable for high resolution thermal imaging. However, the proposed fabrication method is rather impractical and time consuming.

Here we present a novel method for batch fabrication of a scanning microscopy probe by standard micromachining techniques and conventional optical contact lithography. Our probe, shown in Figure 1, has a sharp pyramidal tip composed of freestanding silicon nitride nanowires and is suitable for both thermal and magnetic imaging.
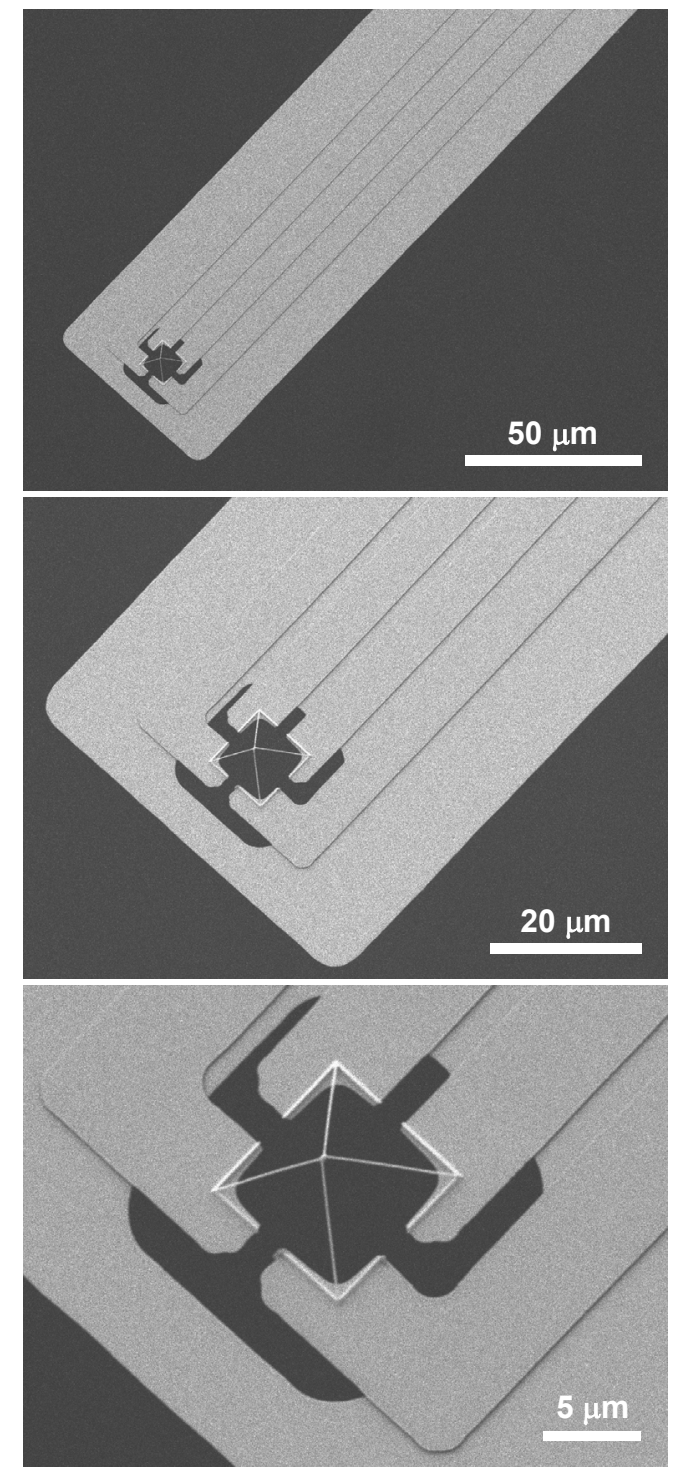

Figure 1: SEM micrographs of a novel scanning probe designed for thermal and magnetic imaging. 


\section{PROBE DESIGN}

A schematic of our scanning probe is shown in Figure 2.

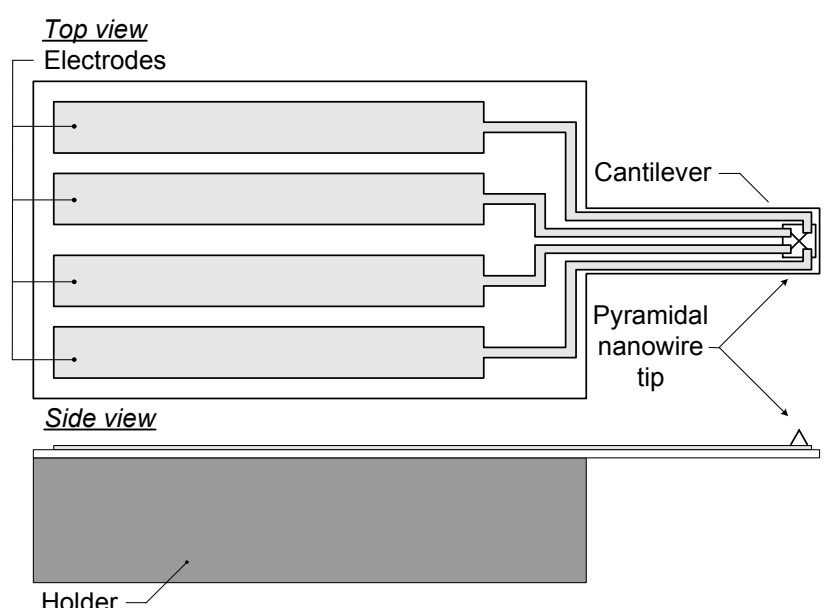

Figure 2: Schematic view of the scanning probe with a pyramidal tip composed of freestanding conductive

The probe features a compliant cantilever attached on a rigid holder. At the end of the cantilever a sharp pyramidal tip is situated. The tip is composed of four freestanding conductive nanowires, which form an electrical cross junction at the apex of the tip. This cross junction is addressable through the electrodes integrated on the cantilever and the probe holder.

\section{MICROFABRICATION}

\section{Corner Lithography}

In order to produce a sharp pyramidal tip composed of freestanding nanowires, corner lithography $[10,11]$ is employed. This method is illustrated in Figure 3.

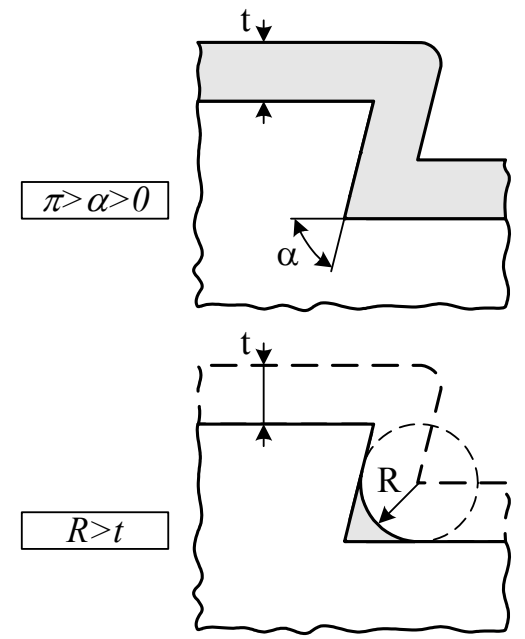

Figure 3: The nanowire pyramid is fabricated by corner lithography. This method employs time-controlled isotropic etching of a conformally deposited layer to form nanometer-sized features in the concave corners.

Corner lithography employs conformal deposition of material onto a prepared template followed by timecontrolled isotropic etching that removes the deposited material from all template surfaces, except the material located in concave corners. The process results in welldefined nanometer scale structures with exact position and spatial arrangement fully determined by the template. The method is compatible with conventional micromachining.

\section{Fabrication process}

Basic fabrication steps of our scanning probe are illustrated in Figure 4.

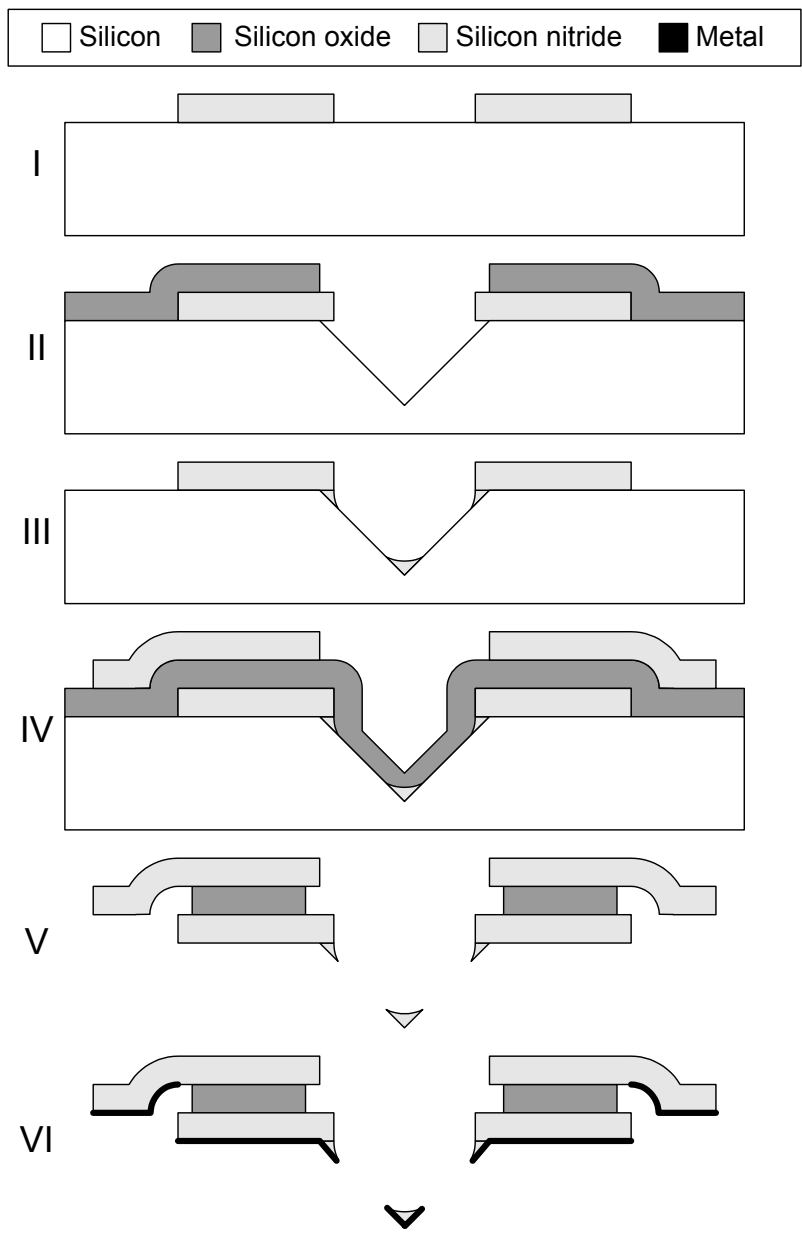

Figure 4: A process of batch fabrication of the scanning probe with a pyramidal nanowire tip by using standard micromachining and conventional optical lithography.

The fabrication process starts on a (100)-oriented standard silicon substrate. On the selected substrate, we deposited a silicon nitride layer by low pressure chemical vapor deposition (LPCVD). The deposited layer is then patterned by reactive ion etching (RIE) to form prospective electrodes (step I). On the patterned surface, a TEOS silicon oxide layer is deposited. In this layer, a rectangular opening is made by BHF etching. Next, a pyramidal cavity is formed by wet anisotropic etching of silicon in $\mathrm{KOH}$ (step II). This cavity serves as a template for the nanowire tip, which is subsequently formed by corner lithography. Corner lithography is performed after removal of the silicon oxide layer. First, we conformally deposited a second silicon nitride layer by LPCVD. Subsequently, we partially removed this layer using timed-control isotropic etching of silicon nitride in concentrated HF (50\%). After the HF etching (step III), the residues of the second silicon nitride layer were only preserved in the sharp corners of the cavity and between 
the sidewalls of the cavity and the first silicon nitride layer. The remaining material has a shape of a nanowire pyramid physically attached to the prospective electrodes that were already formed in the first silicon nitride layer. After corner lithography, we deposited another silicon oxide layer by TEOS deposition. Next, a third silicon nitride layer is deposited by LPCVD and patterned to form a cantilever (step IV). In the next fabrication step which is not shown in Figure 4, the silicon substrate is anodically bonded to a glass substrate in which the probe holders are pre-defined by dicing. After the anodic bonding, the silicon substrate is completely dissolved in a $25 \%$ TMAH solution. Subsequently, the silicon oxide layer is etched in BHF undercutting the prospective electrodes (step $V$ ). In the final step, we made the nanowires and the prospective electrodes conductive by applying a metal coating using evaporation or directional sputter deposition (step VI). The undercut of the prospective electrodes and the three-level structure of the cantilever, shown in Figure 5, ensure proper electrical insulation after the coating. One should note that a wide range of coating materials can be applied.

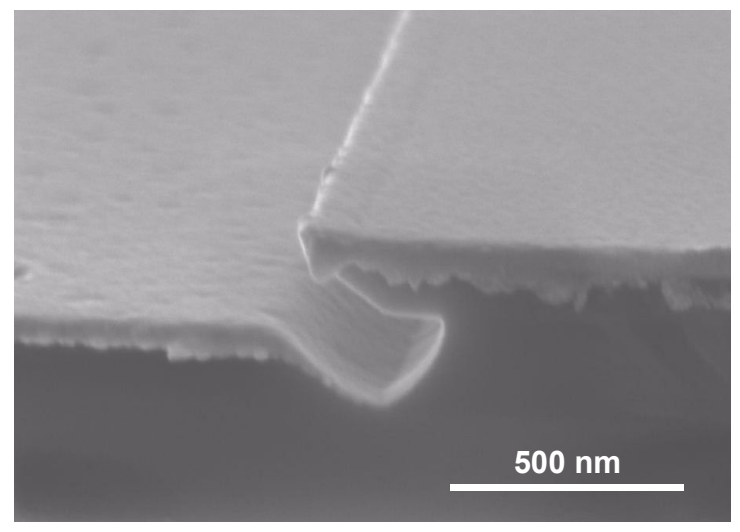

Figure 5: The three-level structure of the probe and an undercut of the prospective electrodes ensure proper electrical insulation after directional deposition of a conductive layer (evaporation or sputtering).

\section{EXPERIMENTAL CHARACTERIZATION}

\section{Fabrication Results}

SEM micrographs of a successfully fabricated first probe prototype are shown in Figure 1. The probe consists of a cantilever, which is $250 \mu \mathrm{m}$ long and $55 \mu \mathrm{m}$ wide. The cantilever has a three-layer structure (silicon nitride/silicon oxide/silicon nitride) with an aggregate thickness of $1.1 \mu \mathrm{m}(350 \mathrm{~nm} / 400 \mathrm{~nm} / 350 \mathrm{~nm})$. The pyramidal tip located at the end of the cantilever is composed of $140 \mathrm{~nm}$ thick and $11 \mu \mathrm{m}$ long silicon nitride wires. The whole probe was metallized by $6 \mathrm{~nm}$ titan (Ti) and $30 \mathrm{~nm}$ gold $(\mathrm{Au})$ layers. The entire probe was integrated on a glass holder with dimensions of $3.4 \mathrm{~mm} \mathrm{x}$ $1.6 \mathrm{~mm}$

\section{Electrical Characterization}

After fabrication, the probe holder was mounted on a printed circuit board and wire-bonded. Four wire-bonds were required to connect all electrodes $(\underline{1}, \underline{2}, \underline{3}$ and $\underline{4})$. After the wire-bonding, we measured the electrical resistance between electrodes by passing a DC current. The resistance was around $1 \mathrm{k} \Omega$ for all possible electrode combinations. In our experiments we could apply a maximum current of $550 \mu \mathrm{A}$ before the breakdown of the nanowires occurred. Given the width of the nanowires of $140 \mathrm{~nm}$ and the thickness of the gold layer of $30 \mathrm{~nm}$, we could calculate a maximum current density of $1.3 \times 10^{7}$ $\mathrm{A} / \mathrm{cm}^{2}$. The order of magnitude of the current density indicates that the breakdown was likely caused by electromigration [12].

The electrical resistance of the nanowires forming the pyramidal tip is a function of temperature. In order to demonstrate the temperature dependence, we have resistively heated the tip by passing a DC current $I_{1-3}$ through one pair of nanowires. The change in electrical

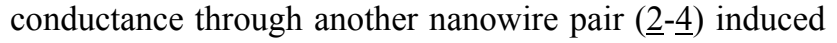
by the heating of the tip was measured using lock-in techniques. The relative change in resistance, which is the ratio between the resistance at a certain level of the heating current and the resistance at room temperature, is shown in Figure 6. As expected, the resistance increases with increasing the heating current.

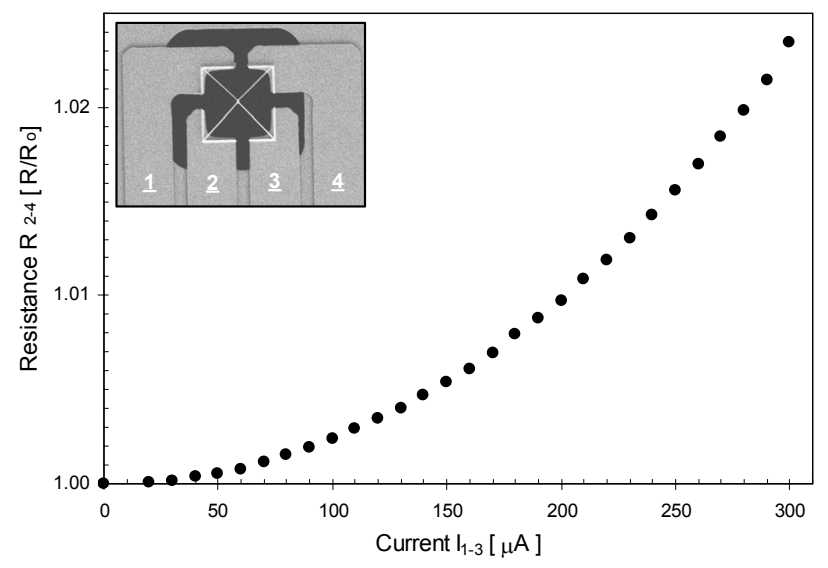

Figure 6: The measured change in electrical resistance $R_{2-4}$ through one pair of nanowires induced by local heating of the tip. The tip is resistively heated by passing a current $I_{1-3}$ through another wire pair.

\section{Magnetic Characterization}

To test the suitability of the probe as a magnetic Hall sensor, we placed it in an external magnetic field. Through one pair of the nanowires $(\underline{1}-\underline{3})$ we passed a current $I_{1-3}$, which flows through the cross junction at the apex of the probe tip. The cross junction serves as a cross Hall element, in which charge carriers are deflected by the Lorenz force induced by the applied magnetic field. Therefore, a Hall voltage can be detected between another electrode pair (2-2 $)$. The voltage $U_{2-4}$ of a cross Hall sensor placed in a static magnetic field $B$ is given by $[7,8]$ :

$$
U_{2-4}=\frac{I_{1-3} B}{n e d}=S_{I} I_{1-3} B
$$

where $n$ is the carrier density, $e$ the elementary charge, $d$ the thickness of the conducting layer and $S_{\mathrm{I}}$ is a current related sensitivity.

Figure 7 shows the Hall voltage $U_{2-4}$ measured as a function of the applied current $I_{1-3}$. The measurements 
were performed at room temperature and with the magnetic fields of $+1.3 \mathrm{~T}$ and $-1.3 \mathrm{~T}$, respectively. As expected from the expression (1), the Hall voltage was linearly proportional to the current. The current related sensitivity $S_{\mathrm{I}}$ of the probe was equal to $1.7 \times 10^{-3} \mathrm{~V} / \mathrm{AT}$. The measured sensitivity was significantly lower (three to four order of magnitudes) compared with Hall devices of similar dimensions realized with semiconductors and semiconductors heterostructures $[7,8]$. The low sensitivity was expected because metal films are generally a poor choice for fabrication of Hall sensors due to their high carrier density and hence very small Hall coefficients. However, it is worth mentioning that at room temperature the resistance noise becomes smaller for mesoscopic $\mathrm{Au}$ Hall devices with respect to their semiconducting counterparts resulting in better signal-to-noise ratio [13]. One should also note that our process is by no means limited to the fabrication of gold Hall probes. Other materials can be used as well. For semiconducting Hall probes, the released probes (step V in Figure 2) should be first coated with a semiconductor. Then, a metallic layer should be applied through a shadow mask to prevent deposition of the metal onto the nanowire tip. In this way, we could create a semiconducting nanowire tip addressable through the metal electrodes.

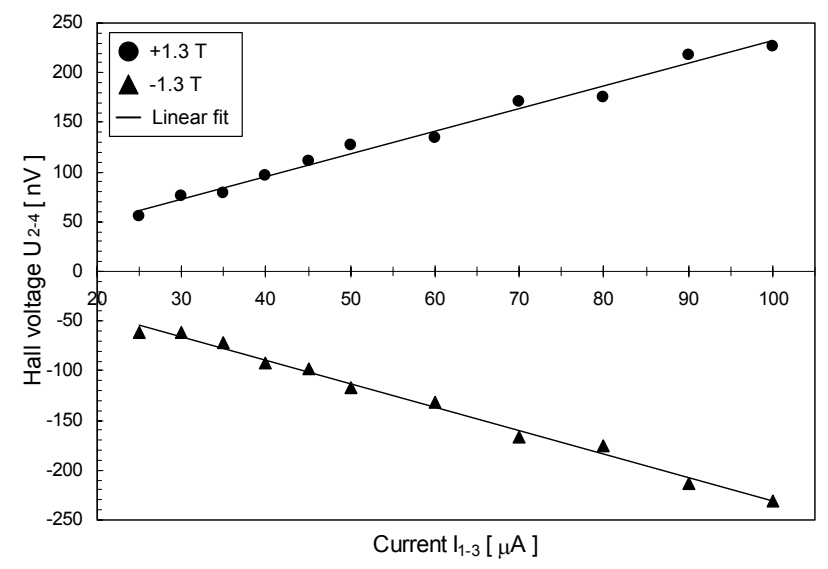

Figure 7: Hall response of the Ti/Au-coated probe at room temperature. The probes was placed in an external magnetic field of $+1.3 T$ and $-1.3 T$, respectively. The offset voltage is removed from the measured signal.

\section{CONCLUSION}

We have devised and fabricated a scanning probe for thermal and magnetic imaging using conventional batch microfabrication methods. Electrical and thermal properties of the probe were experimentally determined demonstrating its proper functioning. In our future research we would employ the scanning probe in an atomic force microscope to measure topographical, thermal and magnetic surface properties. We would also like to apply different coating materials on the nanowire tip in order to improve thermal and/or magnetic properties of the probe (e.g. materials with a higher Hall coefficient for improved sensitivity of a magnetic sensor).

\section{ACKNOWLEDGEMENTS}

The authors would like to thank the staff of the MESA+ Clean Room for their assistance with probe fabrication and P. Linders from Deltamask for the mask processing. The authors gratefully acknowledge J.W. Berenschot and N.R. Tas from the TST group for valuable discussions.

\section{REFERENCES}

[1] H.M. Pollock, A. Hammiche, "Micro-thermal analysis: techniques and applications", J. Phys. D: Appl. Phys. 34, pp. R23-R53, 2001.

[2] B. A. Nelson, W.P. King, "Applications of heated atomic force microscope cantilevers", in "Applied Scanning Probe Methods IV”, Springer Berlin Heidelberg, ISBN: 3540269120, 2006.

[3] H. Zhou, A. Midha, G. Mills, S. Thoms, S.K. Murad, J.M.R. Weaver, "Generic scanned-probe microscope sensors by combined micromachining and electronbeam lithography", J. Vac. Sci. Technol. B 16 (1), pp. 54-58, 1998.

[4] H. Zhou, G. Mills, B.K. Chong, A. Midha, L. Donaldson, J.M.R. Weaver, "Recent progress in the functionalization of atomic force microscope probes using electron-beam nanolithography", J. Vac. Sci. Technol. A 17 (4), pp. 2233-2239, 1999.

[5] K. Edinger, T. Gotszalk, I.W. Rangelow, "Novel high resolution scanning thermal probe", J. Vac. Sci. Technol. B 19 (6), pp. 2856-2860, 2001.

[6] A.M. Chang, H.D. Hallen, L. Harriott, H.F. Hess, H.L. Kao, J. Kwo, R.E. Miller, R. Wolfe, J. van der Ziel, T.Y. Chang, "Scanning Hall probe microscopy", Appl. Phys. Lett. 61, pp.1974-1976, 1992.

[7] G. Boero, M. Demierre, P.A. Besse, R.S. Popovic, "Micro-Hall devices: performance, technologies and applications", Sens. Actuators A 106, pp. 314-320, 2003.

[8] E. Ramsden, "Hall-effect sensors: Theory and applications (Second Edition)", Elsevier, ISBN: 9780-7506-7934-3, 2006.

[9] B.K. Chong, H. Zhou, G. Mills, L. Donaldson, J.M.R. Weaver, "Scanning Hall probe microscopy on an atomic force microscope tip", J. Vac. Sci. Technol. A 19 (4), pp. 1769-1772, 2001.

[10] E. Sarajlic, J.W. Berenschot, G. Krijnen, M.C. Elwenspoek, "Fabrication of 3D nanowire frames by conventional micromachining technology", in Proc. Transducers '05 (Seoul, South Korea), Vol. 1, pp. $27-$ 30, 2005.

[11] J.W. Berenschot, N.R. Tas, H.V. Jansen, M.C. Elwenspoek, "3D-nanomachining using corner lithography", $3^{\text {rd }}$ IEEE Int. Conf. on Nano/Micro Engineered and Molecular Systems (NEMS2008), (Sanya, China), pp. 729-732, 2008.

[12] D.G. Pierce, P.G. Brusius, "Electromigration: A review", Microelectron. Reliab. 37(7), pp. 10531072, 1997.

[13] K.S. Novoselov, S.V. Morozov, S.V. Dubonos, M. Missous, A.O. Volkov, D.A. Christian, A.K. Geim, "Submicron probes for Hall magnetometry over the extended temperature range from helium to room temperature", J. Appl. Phys. 93 (12), pp. 10053100057, 2003. 